\title{
Paracoccidioidomicosis y TBC-MR en portador de $\mathrm{VIH} / \mathrm{VHC}$
}

\author{
Juan Nunura R., Daniela Salazar M., Tania Vásquez L., \\ Sergio Endo G., Alejandrina Rodríguez F. y Rito Zerpa L.
}

\section{Paracoccidioidomicosis and multidrug-resistant tuberculosis (TBC-MDR) in patient coinfected with HIV and hepatitis C}

A case of an adult male patient diagnosed with HIV and Hepatitis C co infection is presented. He had granulomatuos hepatitis and blood smear positive to Paracoccidioides brasiliensis concomitant to the detection of MDR Mycobacterium tuberculosis in sputum further complicated with reactivation of cytomegalovirus (possible pancreatitis and retinitis). Difficulties in diagnostic and therapeutic approach in a patient with multiple infections are reviewed.

Key words: Paracoccidioidomycosis, HIV/AIDS, TBC-MDR, hepatitis C, cytomegalovirus.

Palabras clave: Paracoccidioidomicosis, VIH/SIDA, TBC-MR, hepatitis C, citomegalovirus.

\section{Introducción}

$\mathrm{P}$ aracoccidiomicosis (PCM) es la micosis sistémica de mayor prevalencia en América latina ${ }^{1}$. En el Perú se presenta sólo en las regiones de ceja de selva y selva baja amazónica, debido a que estas zonas de clima tropical reúnen las condiciones ecológicas apropiadas para la supervivencia de Paracoccidioides brasiliensis. Esta micosis no existe en forma autóctona ni en la costa ni en la región andina. Desde 1937 hasta 1971 fueron diagnosticados en el Perú, 147 casos $^{2}$.

El síndrome de inmunodeficiencia adquirida (SIDA) se caracteriza por el desarrollo de varias infecciones oportunistas que incluyen micosis superficiales y profundas. Candida albicans, Criptococcus neoformans e Histoplasma capsulatum son las micosis oportunistas más frecuentemente reportadas ${ }^{3}$.

La co-infección PCM/SIDA es raramente reportada. Hasta el año 2005 fueron publicados 84 casos con dicha asociación $n^{4}$. La tuberculosis (TBC) es una de las infecciones oportunistas (IO) prevalentes en la población infectada con VIH en el Perú ${ }^{5,6}$; asociada a PCM y SIDA se han reportado casos solamente en Brasil ${ }^{7}$. No se ha encontrado reporte similar alguno al caso presentado, en la literatura revisada.

\section{Caso Clínico}

Varón de 54 años de edad, bisexual, enfermero retirado. Natural del Departamento de Huánuco. Historia de viajes frecuentes a su lugar de nacimiento. Pasatiempo: espe- leología. No usuario de drogas. Diagnóstico de infección por VIH en el año 2000. En agosto del 2006 consultó por fiebre y diarrea. Sin medicación profiláctica para IO. El examen físico reveló microadenopatías cervicales e inguinales. IMC: $23 \mathrm{~kg} / \mathrm{m}^{2}$. Exámenes de laboratorio: Reacción inflamatoria en heces: Leucocitos $>100 /$ campo, PMN (99\%), moco (+++), sangre (-). Coprocultivo (-).

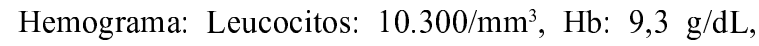
plaquetas: $823.000 / \mathrm{mm}^{3}$. Pruebas hepáticas: Fosfatasa alcalina (FA): $458 \mathrm{U} / \mathrm{L}$, gamma-glutamiltrasferasa (GGT): $158 \mathrm{U} / \mathrm{L}$, transaminasa glutámico oxalacética (TGO): 62 $\mathrm{U} / \mathrm{L}$, albuminemia: $2 \mathrm{~g} \%$. En junio 2006 el recuento de células CD4 fue de 108 células/ $\mathrm{mm}^{3}$ y la carga viral VIH de 1.064543 copias $/ \mathrm{ml}$.

Tras la terapia inicial y remisión de la diarrea, el paciente evolucionó con alzas febriles intermitentes, dolor abdominal tipo cólico difuso, de moderada intensidad. Se amplió el estudio: aspirado y cultivo de médula ósea (-). BAAR en esputo (x3): negativo. Hemocultivos: negativos. TAC abdominal: hepatomegalia y ausencia de cálculos. Anticuerpos para VHC: reactivo. Carga viral VHC: 1.166 $\mathrm{UI} / \mathrm{ml},(3.147$ copias $/ \mathrm{ml})$.

En los exámenes de control se observó un mayor aumento de GGT (397 U/L) y FA: (1.476 U/L). Ante la sospecha de una micosis profunda diseminada se inició terapia con anfotericina $(0,7 \mathrm{mg} / \mathrm{kg} /$ día $)$; la cual recibió en días alternos hasta la dosis acumulada de $600 \mathrm{mg}$, siendo suspendida por elevación de la creatininemia a 1,7 $\mathrm{mg} / \mathrm{dL}$. La fiebre remitió al décimo día de tratamiento, continuándose con fluconazol ev. Resultado de cultivo de BAAR en esputo: crecimiento de una colonia. Se realizó resembrado.
Ministerio de Salud del Perú. Dirección General de Epidemiología (JNR) Universidad Nacional Mayor de San Marcos, Lima, Perú. Sociedad Científica de San Fernando. Facultad de Medicina. (DSM, TVL, SEG, ARF). Instituto de Medicina Tropical "Daniel A. Carrión". (RZL).

Recibido: 11 de julio de 2009 Aceptado: 21 de septiembre de 2010

\section{Correspondencia a:} Juan Nunura Reyes jnunura@dge.gob.pe 
Durante la fase de mantenimiento con fluconazol reapareció la fiebre, por lo que se realizó una punción lumbar obteniéndose los siguientes resultados: glucosa: 33 $\mathrm{mg} / \mathrm{dl}$, ADA: 5,5 U/l, proteínas: 47,4 mg/dl, tinta china (-). Se envió muestra de LCR para cultivo de bacilo de Koch.

El paciente evolucionó con la aparición de nódulos subcutáneos en la pared abdominal se realizó biopsia hepática, cuyo resultado fue hepatitis granulomatosa. BAAR (-).

TAC tórax: infiltrado basal derecho (Figura 1). Ante la probabilidad de una TBC diseminada se decidió empezar tratamiento específico, esquema I del Programa Nacional Control de Tuberculosis (isoniacida + rifampicina + pirazinamida + etambutol) presentando el caso, al séptimo día de tratamiento, reacción adversa a fármacos anti-TBC (RAFA) hepático y aumento de la amilasemia. Fue suspendida toda la medicación.

El paciente evolucionó con tos persistente y disnea por lo que recibió tratamiento para neumonía intrahospitalaria (ciprofloxacina + ceftazidima) observándose una mejoría clínica pero continuó con tos leve.

Fue reiniciado tratamiento anti-TBC con el siguiente esquema: estreptomicina $600 \mathrm{mg} /$ día + etambutol $30 \mathrm{mg} /$ $\mathrm{kg}$-peso/día, continuando con ciprofloxacina $400 \mathrm{mg} /$ día ev dos veces por día. Resultado de cultivo de micobacterias en el LCR: Mycobacterium sp.

Una muestra sanguínea del paciente fue enviada al Instituto de Medicina Tropical de la Universidad Nacional Mayor de San Marcos, encontrándose positivo a P. brasiliensis en el frotis, (Figura 2) con cultivo negativo. La revisión de la biopsia hepática realizada anteriormente, confirmó el compromiso hepático micótico (Figura 3) iniciándose tratamiento con itraconazol $100 \mathrm{mg}$ dos veces al día.

En diciembre 2009, el recuento de CD4 descendió a 19 células $/ \mathrm{mm}^{3}$ y la carga viral para VIH fue de 457.500 copias $/ \mathrm{ml}$.

El paciente continuó con tos leve y dolor abdominal

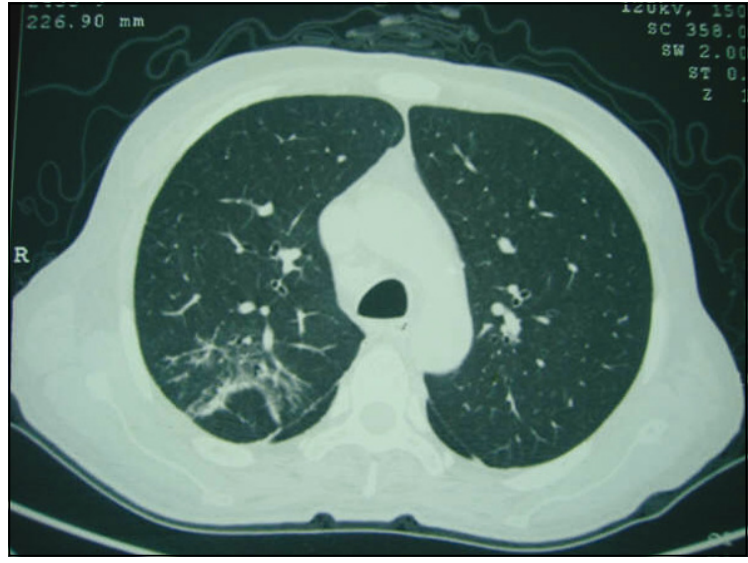

Figura 1. TAC Tórax: Infiltrado crónico inflamatorio basal derecho.

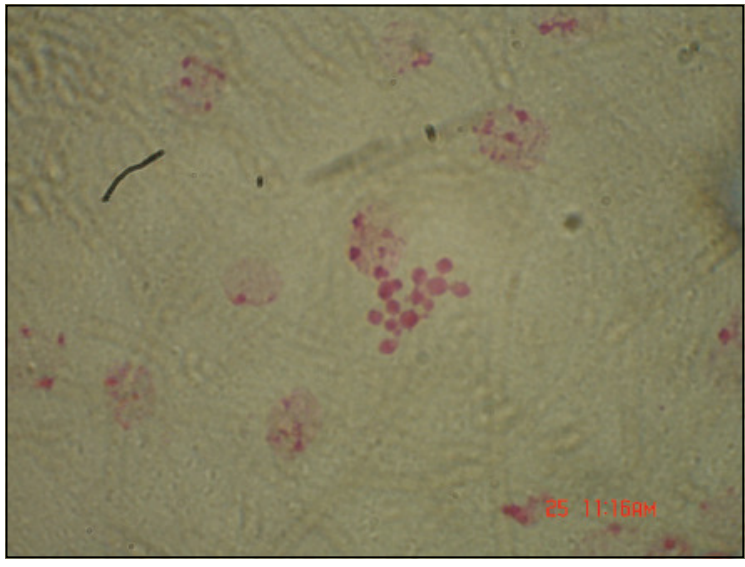

Figura 2. Frotis de sangre: Imágenes típicas en "timón de barco". Coloración Giemsa (40x)
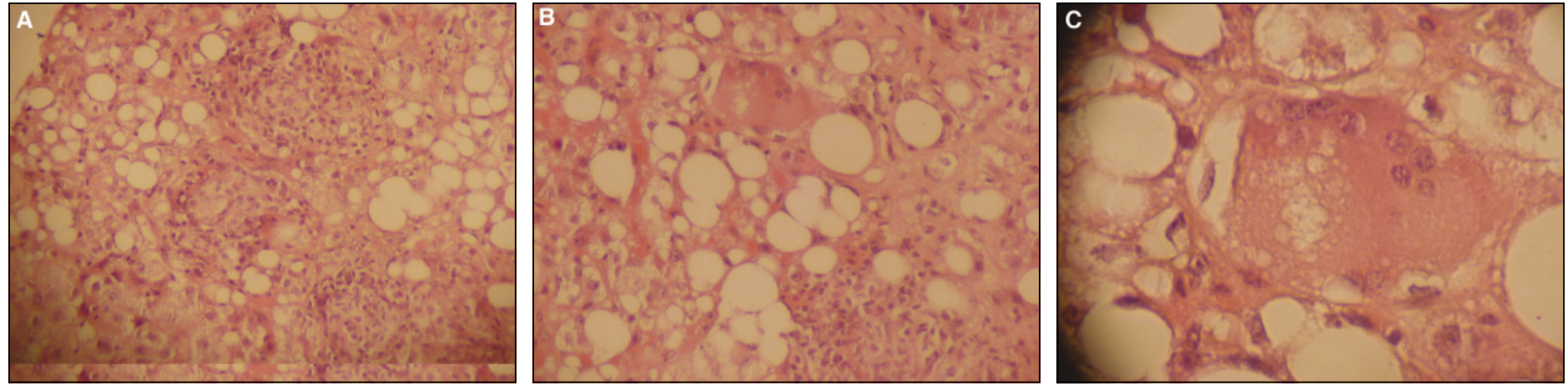

Figura 3. Biopsia hepática. A: Granulomas linfohistiocitarios con células gigantes multinucleadas. H.E. 10x. B: Granuloma (parte inferior) y fagocito (parte superior). H.E. (10x). C: A mayor aumento se observa, célula gigante multinucleada con levaduras del hongo Paracoccidioides brasiliensis en su interior. H.E. (40x). 
esporádico asociado a aumento de amilasemia. Se solicitó serología para citomegalovirus (CMV): IgG 19,1 UI/ml, $\operatorname{IgM} 1,5 \mathrm{UI} / \mathrm{ml}$ (Reactivo $\geq 1,1 \mathrm{UI} / \mathrm{ml})$.

En un control oftalmológico efectuado por uso de dosis plenas de etambutol, se observó retinitis por CMV asintomática. Una segunda serología para $\mathrm{CMV}$, realizada una semana después de la primera, detectó alza de anticuerpos a: IgG 36,5 UI/ml, IgM 2,7 UI/ml. Se inició ganciclovir ev remitiendo el dolor abdominal.

A inicios del año 2007, el paciente presentó febrículas, continuó con tos leve y se evidenció aumento del valor de creatininemia. Por RAFA de tipo renal y sospecha de TBC-MR fue iniciado moxifloxacina $400 \mathrm{mg} /$ día, capreomicina $600 \mathrm{mg}$ tres veces por semana, cicloserina $250 \mathrm{mg}$ /día continuando con etambutol a la dosis de 800 $\mathrm{mg}$ tres veces por semana. Un mes después de iniciado el esquema individualizado anti-TBC, el paciente presentó síntomas compatibles con cuadro de psicosis.

Se decidió iniciar terapia anti-retroviral de gran actividad (TARGA) con esquema 1 del Programa Nacional (zidovudina + lamivudina: 1 tableta en las mañanas y zidovudina + lamivudina + nevirapina: 1 tableta en la noche) presentando acentuación de síntomas psicóticos y falla hepática. Se continuó con zidovudina + lamivudina: 1 tableta dos veces al día. El paciente persistió con síntomas psicóticos acentuados y falleció por insuficiencia hepática aguda tras la administración de medicación antipsicótica.

El resultado de la sensibilidad de resembrado del cultivo de BK en esputo fue resistente a isoniacida y rifampicina. No se realizó necropsia.

\section{Discusión}

Paracoccidioidomicosis (PCM) es una infección producida por el hongo $P$. brasiliensis, que se restringe a Latinoamérica ${ }^{8}$. En el Perú existen pocos trabajos realizados respecto a esta micosis. Burstein ${ }^{2}$ realizó una revisión de 111 casos desde el año 1937, reportando que los casos fueron procedentes con mayor frecuencia de las zonas selváticas de los Departamentos de Junín y Huanuco. Encontró además que las edades entre 31 y 60 años de edad fue el grupo más afectado.

Paracoccidioidomicosis tiene varios factores de riesgo asociados a su adquisición y desarrollo: procedencia de zona endémica, sexo masculino $y$, en pacientes con infección por $\mathrm{VIH}$; la falta de profilaxis con cotrimoxazol'; todos ellos estaban presentes en este caso.

La prevalencia estimada de PCM entre personas infectadas con el VIH en algunos estados de Brasil es relativamente baja cuando se compara con otras micosis tales como histoplasmosis y criptococosis ${ }^{10}$. Hasta marzo del 2003 en dicho país fueron publicados 104 casos de PCM en pacientes con VIH/SIDA, lo cual contrasta con los 2.500 casos de SIDA e histoplasmosis ${ }^{11}$. Generalmente los pacientes co-infectados $\mathrm{PCM} / \mathrm{VIH}$, tienen un recuento de linfocitos T CD4 inferior a 200 células $/ \mathrm{mm}^{312}$. La actual baja incidencia de PCM en pacientes con infección por VIH puede atribuirse al uso extendido de TARGA y al uso de cotrimoxazol como profilaxis, ya que ésta es un fármaco efectivo contra $\mathrm{PCM}^{13}$.

En inmunocomprometidos con $\mathrm{PCM}$, la reactivación lleva hacia una enfermedad mixta diseminada ${ }^{3}$. En el caso presentado, el diagnóstico etiológico se basó en el hallazgo de levaduras de $P$. brasiliensis en sangre más un compromiso hepático granulomatoso micótico. Se considera al examen directo micológico como el estándar de oro para el diagnóstico de $\mathrm{PCM}^{14}$; la confirmación de un caso probable puede ser realizada a través de este modo ${ }^{15}$ aunque su detección en sangre a través del frotis no es lo habitual ${ }^{16}$.

Es probable que el cultivo negativo para PCM que obtuvimos haya sido por el reinicio de la profilaxis con cotrimoxazol en los días previos.

El caso presentó una recaída con el uso de fluconazol debido a que el anti-fungico de elección en PCM es itraconazol ${ }^{17}$.

En el Perú, los pacientes infectados con el VIH están en riesgo de contraer y morir por $M$. tuberculosis, siendo la TBC multiresistente (MR) un problema emergente. La TBC-MR en el Perú se asocia a tratamiento inadecuado, pobre adherencia al tratamiento e infección por $\mathrm{VIH}^{18,19}$. En el caso presentado además de la infección por VIH se agregó como factor de riesgo, una estancia hospitalaria prolongada.

El crecimiento de una colonia en el cultivo de BK en esputo, como ocurrió en nuestro paciente, es indicativo de TBC pulmonar, dado que el crecimiento de $M$. tuberculosis no se da por contaminación. La baciloscopia en esputo fue negativa, lo cual puede ser explicado por su condición de inmunosuprimido siendo común que en pacientes con infección por VIH se manifieste un cuadro clínico atípico y sea común la diseminación extrapulmonar, así como los eventos adversos relacionados al tratamiento ${ }^{20}$.

Cerca de un tercio de los pacientes infectados con VIH tienen hepatitis C crónica, llegando esta tasa hasta 75\% en usuarios de drogas $\mathrm{ev}^{21}$. En la actualidad existe una mayor evidencia de transmisión vía sexual del $\mathrm{VHC}^{22,23}$. El caso presentado tuvo antecedentes de exposición sexual (bisexual) y posiblemente parenteral (enfermero que había trabajado en un servicio quirúrgico).

Los pacientes co-infectados por VIH y VHC tienen una rápida progresión de la hepatitis, con aumento de la carga viral y daño hepático grave lo cual aumenta el riesgo de muerte por enfermedad hepática ${ }^{24}$. Sin embargo, en el caso descrito la disfunción hepática fue multifactorial. (Inicialmente fue tóxica por isoiacida y/o rifampicina; finalmente por nevirapina y fármaco antipsicótico). 
La recomendación en pacientes co-infectados con VIH/ VHC es empezar TARGA ${ }^{25}$, aunque el VHC es a su vez, un fuerte predictor para el desarrollo de hepatotoxicidad por TARGA $^{26,27}$. Se ha reportado que la enfermedad crónica hepática más frecuente en pacientes co-infectados por PCM y VIH es causada por el VHC ${ }^{28}$.

Durante la evolución, el paciente presentó elevación de la amilasemia concomitante al inicio de tratamiento anti $\mathrm{TBC}$; sin embargo, al suspenderse dicha terapia permanecieron elevados los controles de amilasemia y el dolor abdominal, lo cual sugirió otra etiología.

El paciente tuvo serología de reactivación para CMV concomitante a la caída de células CD4 a $<50$ células/ $\mathrm{mm}^{3}$. Además se diagnóstico retinitis por $\mathrm{CMV}$ asintomática por lo que se consideró al CMV como etiología del dolor abdominal y del aumento de la amilasemia. El CMV es el microorganismo que más frecuentemente afecta el páncreas en pacientes infectados por VIH, siendo el compromiso a menudo silente y de reconocimiento difícil, por lo que no es sospechado; su diagnóstico generalmente es post mortem ${ }^{29-31}$. En nuestro caso no se pudo confirmar el compromiso pancreático por CMV dado que no se realizó necropsia.

\section{Resumen}

Reportamos el caso de un varón de 54 años portador de VHC y VIH estadio SIDA quien tuvo hepatitis granulomatosa y frotis de sangre positivo a Paracoccidioides brasiliensis concomitante al hallazgo de Mycobacterium tuberculosis multiresistente en esputo, que evolucionó con reactivación de citomegalovirus (pancreatitis probable y retinitis). Se describen las dificultades diagnósticas y terapéuticas en un paciente con múltiples infecciones.

\section{Referencias}

1.- Martínez R. Etioepidemiologia e ecologia da Blastomicose Sul-Americana (Paracoccidioidomicose). Veronessi R, Focaccia R, Editores. Tratado de Infectologia, $2^{a}$ Ed. 2004. São Paulo Brasil. Editorial Atheneu. p. 1101-3.

2.- Burstein Z. Revisión. Aspectos clínicos de la Blastomicosis Sudamericana (Paracoccidioidomicosis) en el Perú. Rev Peru Med Exp Salud Pública 2002; 19 (1): 43-7

3.- Benard G, Duarte AJ. Paracoccidioidomycosis: a model for evaluation of the effects of human immunodeficiency virus infection on the natural history of endemic tropical diseases. Clin Infect Dis 2000; 31 (4): 1032-9

4.- Montani Caseiro M, Etzel A, Soares M C B, Olavo Pinto da Costa S. Septicemia caused by Paracoccidioides brasiliensis (Lutz, 1908) as the cause of death of an AIDS patient from Santos, São Paulo State, Brazil - A nonendemic area. Rev Inst Med Trop S Paulo 2005; 47 (4): 209-11.

5.- Alvarezcano J, López J, Soria J, Vásquez R. Epidemiología de los casos incidentes con infección VIH en el Hospital Nacional Dos de Mayo 2006 En: Libro de resúmenes del X Congreso Peruano de Enfermedades Infecciosas y Tropicales. Lima. Sociedad Peruana de Enfermedades Infecciosas y Tropicales. 2007. Abstract 16, Sección 2 ETS y VIH/SIDA.

6.- Nunura J, Mendo F. Perfil clínico de los pacientes VIH hospitalizados en la era HAART. Rev Soc Peru Enf Infec Trop 2004; 3 (1): 18-22.

7.- Nogueira S, Caiuby J, Vasconcelos V, Halpern M, Gouveia C, Thorpe B, et al. Paraccoccidioidomycosis and tuberculosis in
AIDS patients: Report of two cases in Brazil. Intern J Infect Dis 1998; 2 (3): 168-72.

8.- Restrepo A, Restrepo M, Botero D, Trujillo H, Leiderman E, Robledo J, et al. Fundamentos de Medicina Enfermedades Infecciosas. 4ta Ed. 1991. Medellín Corporación para Investigaciones Biológicas, p. 75.

9.- Goldani L Z, Sugar A M. Paracoccidioidomycosis and AIDS: an overview. Clin Infec Dis 1995; 21: S1275-8.

10.- Brunaldi M O, Rezende R E F, Zucoloto S, García S B, Módena J L P, Machado A A. Co-infection with paracoccidioidomycosis and human immunodeficiency virus: Report of a case with esophageal involvement. Am J Trop Med Hyg 2010; 82 (6): 1099-101.

11.- Botelho A, Aires E, Moreira I, Pappalardo M, Ferreira A, Bozczowski I. Paracoccidioidomicose disseminada em paciente com AIDS Em: Livro de resumos do XLI Congresso da Sociedade Brasileira de Medicina Tropical 2005. Abstract AI 27 p. 10

12.- Tobon A, Orozco B, Estrada S, Jaramillo E, Bedout C, Arango M, et al. Paracoccidioidomycosis and AIDS: report of the first two Colombian cases. Rev Inst Med Trop Sao Paulo 1998; 40 (6): 377-81.

13.- Lacaz C S, Minami O S. Ação in vitro da sulfamida Ro 4-4393 sôbre o Paracoccidioides brasiliensis. Resultados preliminares Hospital (Río) 1963; 64: 83-7.

14.- Vargas J, Vargas R. Revisión. Paracoccidioidomicosis. Rev Enferm Infecc Trop 2009; 1: 49-56. [Fecha de acceso: 01 de julio 2010] Disponible en: http://www.revistasbolivianas. org.bo/pdf/reit/v1n1/v ln la12.pdf

15.- Shikanai-Yasud M A, Telles Filho F Q, Pôncio R, Lopes A, Moretti M L. Consenso $\mathrm{em}$ paracoccidioidomicose. Guideliness in paracoccidioidomycosis. Rev Soc Bras Med Trop 2006; 39 (3): 297-310.

16.- Burstein Z. Aporte al diagnóstico de las micosis humanas en el Perú. (Tesis Doctoral). Lima: Facultad de Medicina, Universidad Nacional Mayor de San Marcos; 1970.

17.- Forneron A. Paracoccidioidomicosis: tratamiento con itraconazol. Mem Inst Investig Cienc Salud 2006; 4 (2): 7-11.

18.- Campos P E, Suárez P G, Sánchez J, Zavala D, Arevalo J, Ticona E, et al. Multidrug-resistant Mycobacterium tuberculosis in HIV-infected persons, Peru. Emerg Infect Dis 2003; 9: 1571-8.

19.- Bonilla C A, Crossa A, Jave H O, Mitnick C D, Jamanca R B, Herrera C, et al. Management of extensively drug-resistant tuberculosis in Peru: Cure is possible. PLoS ONE 2008; 13; 3 (8): e2957.

20.- Kavai V, Soto G, Gilman R H, Bautista C T, Caviedes L, Huarato L, et al. Tuberculosis mortality, drug resistance and infectiousness in patients with and without HIV infection in Peru. Am J Trop Med Hyg 2006; 75 (6): 1027-33.

21.- Focaccia R. Tratado de hepatites virais $1^{a}$ Ed., 2003. São Paulo. Brasil Edit Atheneup. Capítulo 17.2 Coinfeccão HIV/HCV. Soriano V, garcía Samaniego J, Puoti M. 693.

22.- Soto B, Rodrigo L, García-Bengoechea M, Sánchez-Quijano A, Riestra S, Arenas J I, et al. Heterosexual transmission of hepatitis $\mathrm{C}$ virus and the possible role of coexistent human immunodeficiency virus infection in the index case. A multicentre study of 423 pairings. J Intern Med 1994; 236: 515-9.

23.- Thomas D L, Zenilman J M, Alter H J, Shih J W, Galai N, Carella A V, et al. Sexual transmission of hepatitis $\mathrm{C}$ virus among patients attending sexually transmitted diseases clinics in 
Baltimore-an analysis of 309 sex partnerships. J Infect Dis 1995; 171: 768-75.

24.- Graham C S, Baden L R, Yu E, Mrus J M, Carnie J, Heeren T, et al. Influence of human immunodeficiency virus infection on the course of hepatitis c virus infection: a meta-analysis. Clin Infect Dis 2001; 33: 562-9.

25.- Hoffmann C, Rockstroh J, Kamps B. HIV Medicine $15^{\mathrm{a}}$ Ed. 2007. Hamburg Bonn Paris Flying Publisher. Content 18. HIV and HBV/ HCV coinfections. Wasmuth Jch and Rockstroh J. p. 543.

26.- Brinker M D, Wit F W, Dillen W V, Jurriaans S, Weel J, Leeuwen V, et al. Hepatitis B and C virus co-infection and the risk for hepatotoxicity of highly active antiretroviral therapy in HIV-1 infection. AIDS 2000; 14: 2895-902.

27.- Sulkowski M, Thomas DL, Chaisson RE, Moore RD. Hepatotoxicity associated with antiretroviral therapy in adults infected with human immunodeficiency virus and the role of hepatitis C or B virus infection. JAMA 2000; 283: 74-80.

28.- Loro K, Macahado A, Martínez R. Paracoccidioidomycosis in patients infected with and not infected with human immunodeficiency virus: A case control study Am J Trop Med Hyg 2009; 80 (3):
359-66.

29.- Wolf P, Reiser JR, Fellow JE, Haghighi P. Pancreatitis in patients with AIDS presumptively due to CMV J. Clin Lab Anal 1989; 3: 152-5.

30.- González-Reimers E, Santolaria-Fernández F, Gómez-Srvent J. Cytomegalovirus-associated pancreatitis in acquired immunodeficiency syndrome. HPB Surgery 1992; 5: 181-4.

31.- Wilcox C M, Forsmark C E, Grendell J H, Darragh T M, Cedió J P. Cytomegalovirus associated acute pancreatic disease in patients with acquired immunodeficiency syndrome. Gastroenterology 1990; 99: 263-7. 